\title{
Adequate Models for Multiprocessor Stochastic Systems
}

\author{
Nadwa S. Younis \\ Department of Mathematics / College of Education \\ University of Mosul
}

Received

02 / 05 / 2011
Accepted

$05 / 10 / 2011$

\begin{abstract}
الخلاصة
في هذا البحث ، تم تقديم نموذجين للأنظمة العشوائية المتعد دة العمليات ـ الأول هو

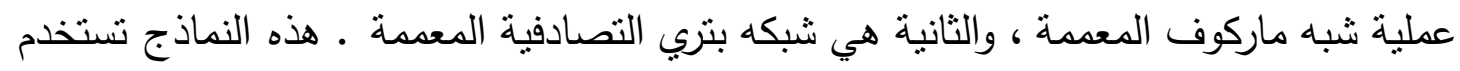

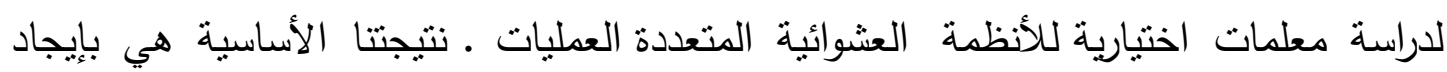

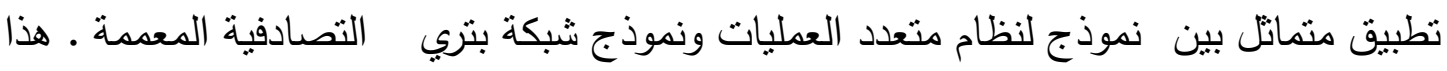
التطبيق المتماتل بغطي الهيكلية الحركية الأساسية للأنظمة العشوائية المتعددة العمليات.
\end{abstract}

\begin{abstract}
In this paper, two models are presented for Multiprocessor Stochastic Systems (MSS). The first one is the Generalized Semi-Markov Process (GSMP), the second is the Generalized Stochastic Petri Net (GSPN). These models are used to study performance arbitrary parameters of (MSS). Our main result is by constructing an isomorphism mapping between (MSS) model and (GSPN) model. This isomorphism captures the essential dynamical structure of (MSS).
\end{abstract}

\section{INTRODUCTION}

Markov modulated processes are very often used to model different arrival processes of Multiprocessor Stochastic System (MSS). The major problem of each Markov model is the size of the state space which grows exponentially in the system complexity. Only some algorithms could by applied to that model. The other important mathematical model used to analyze Multiprocessor Stochastic System is the Generalized Stochastic Petri Net (GSPN) model which is an abstract formal model of information flow and is used to represent concurrency, synchronization, and communication in complex system. [9] [13] [11] [6]. 
In this paper we divided the work as follows:

In section 2 we define and analyze the Multiprocessor Stochastic System with an example, while in section 3 we define and discuss the (GSPN) model also with an example. Finally, in section 4 we construct an isomorphism from a mathematical framework of (GSMP) model for (MSS) into a mathematical framework of (GSPN) model for (MSS), and in the end of this section we give conclusions.

\section{ANALYSIS OF MULTIPROCESSOR STOCHASTIC SYSTEMS}

Now we will present a multiprocessor stochastic system with a common storage, $\mathrm{N}$ different processors and only one server [4]. The different job arrivals and the characterization of the system are presented using (MSS) model and Markov modulated processes. A simple system specification looks as follows:

Each of the $\mathrm{N}$ processors, which are connected with one common storage, sends requests to the common memory using the server
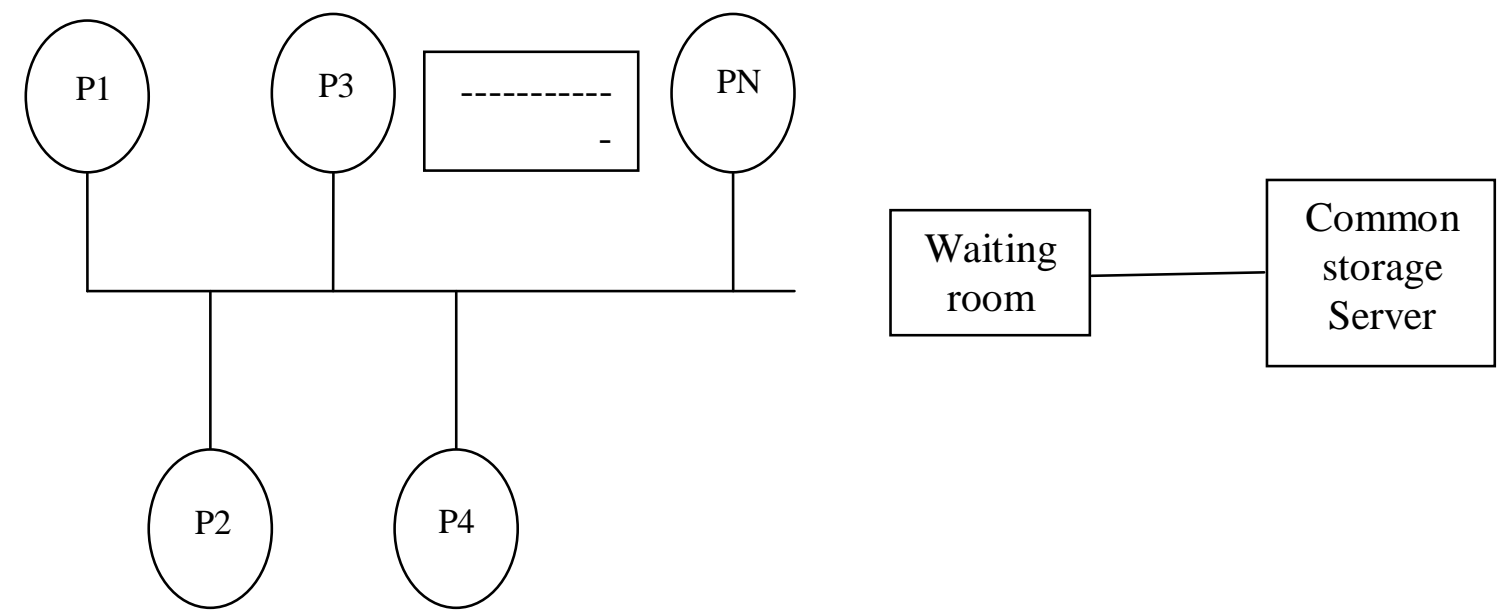

Figure 1. Shows a simple model of Multiprocessor Stochastic System

A simple submodel of this Multiprocessor Stochastic System follows:[15]

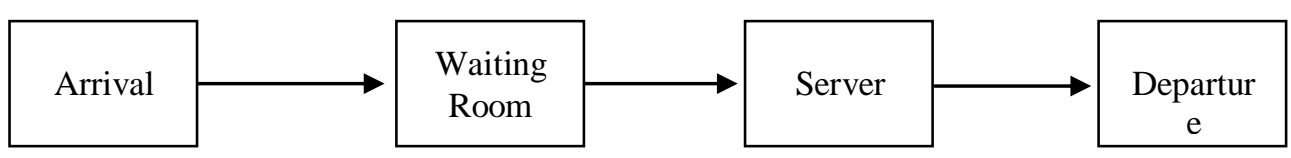

Figure 2. Shows single - server queuing system

If for example in a (MSS) the request generation time is assumed to be exponentially distributed as well as the server allocation time. Also, all random variables are assumed to be stochastically independent and each processor is characterized by its own arrival and service rate. In this case some type of Markov processes models are used to analyzed the stochastic behavior of the system [5]. 
Since no computationally treble analytical results are available for calculating the exact value of mean value of the waiting time for single or Multiprocessor Stochastic System, simulation is a natural recourse [2]. Recently in [11] they present a method to obtain performance parameters from (GSPN) model to be able to analyze the stochastic behavior of (UML) system (which is a special case of (MSS) system). They derived Continuous Time Markov Chain (CTMC) from (GSPN) and they used Markov chain theory to obtain the performance parameters.

Now, we can present a mathematical framework of Markov processes that may be used to analyze the stochastic behavior of (MSS).

\section{Theorem 2.1: [10] [8]}

Let $\{X(t): t \geq 0\}$ be a sequence of random variables with state space $\mathrm{S}$. Assume that:

1- There exist a sequence $\left\{T_{\mathrm{k}}: \mathrm{k} \geq 0\right\}$ of stopping times such that $\left\{T_{\mathrm{k}+1}-T_{\mathrm{k}}: \mathrm{k} \geq 0\right\}$ are independent and identically distributed.

2 - for every sequence of times $0<\mathrm{t}_{1}<\mathrm{t}_{2} \ldots<\mathrm{t}_{\mathrm{m}}(\mathrm{m}>1)$ and $\mathrm{k} \geq 0$, the random vectors $\left\{X\left(\mathrm{t}_{1}\right), \ldots, X\left(\mathrm{t}_{\mathrm{m}}\right)\right\}$ and $\left\{X\left(T_{\mathrm{k}}+\mathrm{t}_{1}\right), \ldots, X\left(T_{\mathrm{k}}+\mathrm{t}_{\mathrm{m}}\right)\right\}$ have the same distribution and the processes $\left\{\mathrm{X}(\mathrm{t}): \mathrm{t} \leq \mathrm{T}_{\mathrm{k}}\right\}$ and $\left\{\mathrm{X}\left(T_{\mathrm{k}}+\mathrm{t}\right): \mathrm{t} \geq 0\right\}$ are independent .

3- $\quad X(t)=X_{\mathrm{n}}$ on $T_{\mathrm{n}}<t \leq T_{\mathrm{n}+1}$.

4- $\quad \operatorname{pr}\left(X_{\mathrm{n}+1}=j, T_{\mathrm{n}+1}-T_{\mathrm{n}} \leq t / X_{0}, \ldots, X_{\mathrm{n}} ; T_{0}, \ldots, T_{\mathrm{n}}\right)=\operatorname{pr}\left(X_{\mathrm{n}+1}=j\right.$ $\left.T_{\mathrm{n}+1}-T_{\mathrm{n}} \leq t / X_{\mathrm{n}}\right)$ for all $\mathrm{n}=0,1, \ldots, j \in \mathrm{S}$, and $t \geq 0$.

Then the process

$\{X(\mathrm{t}): t \geq 0\}$ (or $\left.\left\{\left(X_{\mathrm{n}}, T_{\mathrm{n}}\right): n \in N\right\}\right)$ is a Semi-Markov process.

Collaray 2.2: If in Theorem $2.1 T_{\mathrm{n}+1}-T_{\mathrm{n}}=1$ for all $\mathrm{n} \geq 0$. Then $\{X(\mathrm{t}): \mathrm{t} \geq 0\}$ is a discrete time Markov Chain .

Collaray 2.3: If in Theorem 2.1 $T_{\mathrm{n}+1}-T_{\mathrm{n}}$ has an exponential distribution whose parameter, only depend, on the value of $X_{\mathrm{n}}$. Then $\{X(t): t \geq 0\}$ is a Continuous Time Markov Chain (CTMC).

A Generalized Semi-Markov Process (GSMP) were introduced by Schassberger in [14]. A GSMP moves from state to state with the destination and duration of each transition depending on which of several possible events associated with the occupied state occurs first. We give a formula of the following theorem from [14], [2] and [4].

Theorem 2.4 : Let $\{X(t): t \geq 0\}$ be a sequence of random variables with state space $\mathrm{S}$. Let $E=\left\{e_{1}, \ldots, e_{\mathrm{m}}\right\}$ be a finite set of events. Assume that:

1- For $s \in S, E(s)$ denotes the set of events that can occur when the process is in the state $\mathrm{s}$ and $p(s, s, e)$ is a probability of new state $\mathrm{s}$ by the evente. 
2- there exists a sequence $\left\{\left(s_{\mathrm{n}}, c_{\mathrm{n}}\right): \mathrm{n}=0,1, \ldots\right\}$ of state clock time such that $\left(s_{\mathrm{i}}, c_{\mathrm{i}}\right)$ and $\left(s_{\mathrm{j}}, c_{\mathrm{j}}\right) i \neq j$ are independent and identically distributed where $c_{\mathrm{n}}$ is the clock time reading of event associated with state $s_{\mathrm{n}}$.

3- $\theta_{0}=0$ and $\theta_{\mathrm{n}}=\sum_{k=0}^{n-1} t^{*}\left(s_{\mathrm{k}}, c_{\mathrm{k}}\right)$ is the nth time at which the process makes a state transition.

4- $\operatorname{pr}\left(\sup _{n \geq 1} \theta_{\mathrm{n}}=\infty /\left(s_{0}, c_{0}\right)\right)=1$ a.s.

5- $X(t)=S_{N(t)}, N(t)=\max \left\{n \geq 0 ; \theta_{\mathrm{n}} \leq t\right\}$. then $\{X(t): t \geq 0\}$ is a GSMP.

\section{APPLICATIONS OF GSPN MODEL}

Generalized Stochastic Petri Nets (GSPN) have recently emerged as principal performance modeling tool for distributed systems such as multiprocessor, local area networks, and automated manufacturing systems. In fact, there are many types of PN models [1]. The most important useful nowadays is the (GSPN) model. A (GSPN) have been used to analyze the performance of hardware and software systems. Note that the hardware and software systems that have been modeled successfully with GSPN $^{\text {, s }}$ include communication protocols, parallel programs, multiprocessor memory caches and distributed databases. Also $\mathrm{GSPN}^{\mathrm{s}}$ are a popular graphical modeling formalism for investigating the qualitative and qualitative properties of concurrent systems [16].

Now, we can present the mathematical framework of a GSPN that may be also used to analyze the stochastic behavior of MSS.

GSPN are extensions of Place - Transition nets, which are untimed Petri nets with no transition firing delays.

A Place - Transition net is formally defined as [3]:

\section{Definition 3.1:}

A Place - Transition net is a 5 - tuple $P N=\left(P T, I, I^{+}, M_{0}\right)$ where:

1- $P=\left\{p_{1}, \ldots, p_{\mathrm{n}}\right\}$ is a finite and non - empty set of places.

2- $T=\left\{t_{1}, \ldots, t_{\mathrm{m}}\right\}$ is a finite and non - empty set of transitions.

3- $P \cap T=\phi$.

4- $I, I^{+}: P \times T \longrightarrow M_{0}$ are the backward and forward incidence functions respectively. If $\mathrm{I}^{-}(p, t)>0$, an arc leads from place $\mathrm{p}$ to transition $\mathrm{t}$, and if $I^{+}(p, t)>0$ then an arc leads from transition $\mathrm{t}$ to place $\mathrm{p}$.

5- $M_{0}: P \longrightarrow{ }_{0}$ is the initial marking defining the initial number of tokens on every place.

A marking is a vector of integers representing the number of tokens on each place in a Petri net. The set of all markings that are reachable from the initial marking $M_{0}$ is known as the state space or reachability set of the Petri net, and is denoted by $R\left(M_{0}\right)$. The connections between 
markings in the reachability set from the reachability graph. Formally, if the firing of a transition that is enabled in marking $M_{\mathrm{i}}$ results in marking $M_{j}$, then the reachability graph contains a directed are from marking $M_{\mathrm{i}}$ to marking $M_{j}$.

GSPN $^{\mathrm{s}}$ are timed extensions of Place - Transition nets with two types of transitions: immediate transitions and timed transitions. Once enabled, immediate transitions fire in zero time, while timed transitions fire after an exponentially distributed firing delay. Firing of immediate transitions therefore has priority over the firing of timed transitions.

The formal definition of a GSPN is as follows:

\section{Theorem 3.2: [3]}

A GSPN is a 4 - tuple GSPN $=\left(P N, T_{1}, T_{2}, W\right)$ where:

1- $P N=\left(P T, I, I^{+}, M_{0}\right)$ is the underlying Place - Transition net.

2- $\quad T_{1} \subseteq T$ is the set of timed transitions, $T_{1} \neq \phi$,

3- $T_{2} \subset T$ denotes the set of immediate transitions, $T_{1} \cap T_{2}=\phi$, $T=T_{1} \cup T_{2}$.

4- $\quad W=\left(w_{1}, \ldots, w|T|\right)$ is an array whose entry $w_{\mathrm{i}}$ is either

- a (possibly marking dependent) rate $0 \mathrm{IR}^{+}$of an exponential distribution specifying the firing delay. When transition $t_{\mathrm{i}}$ is a timed transition, i.e. $t_{\mathrm{i}} \in T_{1}$ Or

- a (possibly marking dependent) weight $0 \quad \mathrm{IR}^{+}$specifying the relative firing frequency, when transition is an immediate transition, i.e. $t_{\mathrm{i}} \in T_{2}$.

The reachability graph of a GSPN contains two types of markings.

A vanishing marking is one in which an immediate transition is enabled. The sojourn time in such markings is zero. A tangible marking is one which enables only timed transitions. The sojourn time in such markings is exponentially distributed. We denote the set of reachable vanishing markings by $\gamma$ and the set of reachable tangible markings by $\tau$.

We define $p_{\mathrm{ij}}$ to be the probability that $j$ is the next marking entered after marking $\mathrm{i}, \mu_{\mathrm{i}} M_{i}^{-1}$ to be the man sojourn time in marking $i$ and, for $i 0 \tau, q_{\mathrm{ij}}=\mu_{i} p_{\mathrm{ij}}$;i.e. $q_{\mathrm{ij}}$ is the instantaneous transition rate into marking $j$ from marking $i$.

The following example is an application model for MSS for more details see [12]. 


\section{Example 3.3: [12]}

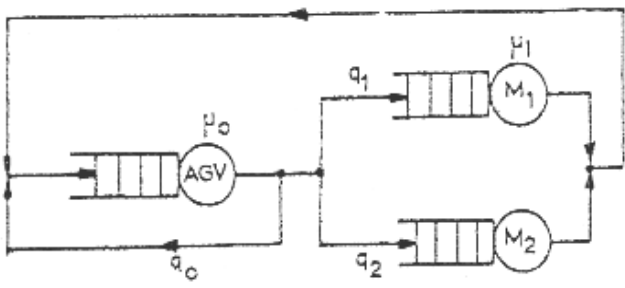

Figurofr A closed Queveutng Network Model.

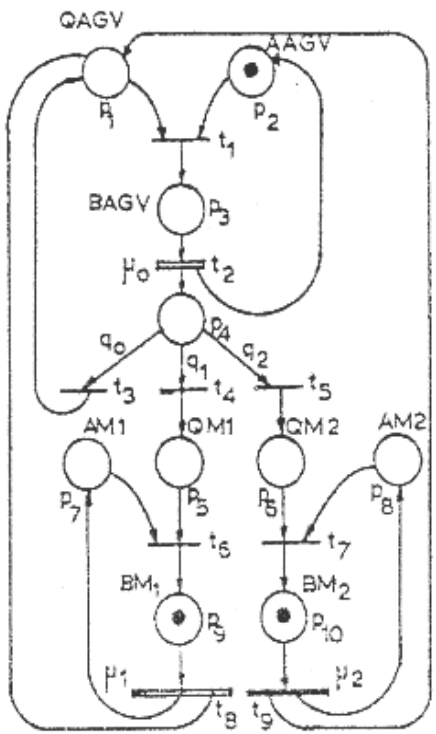

Fagure 2. GSFi Wodel of the Queveing Network in Big. 1.

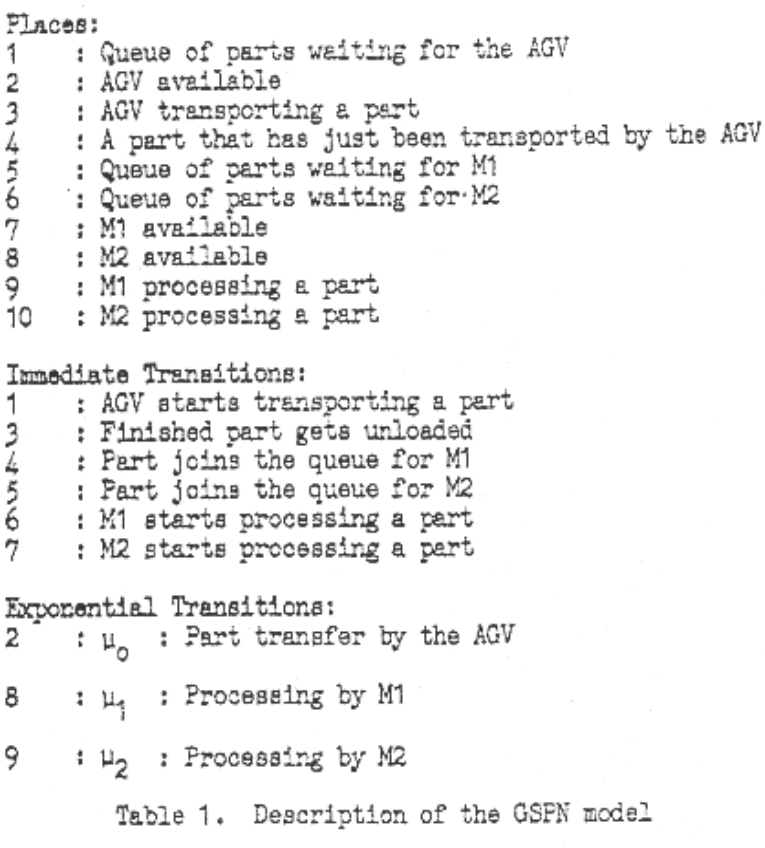

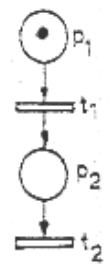

3(a) Sequentigl Axecution

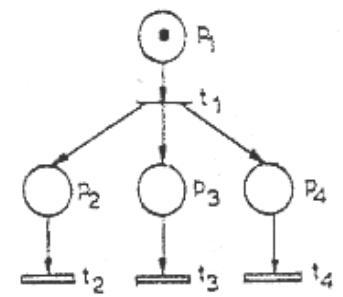

$3(b)$ Concurrency

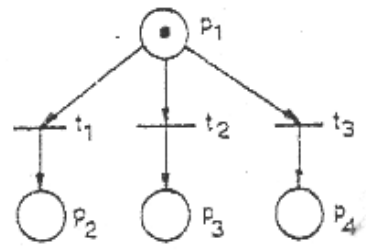

3ic) Conflict

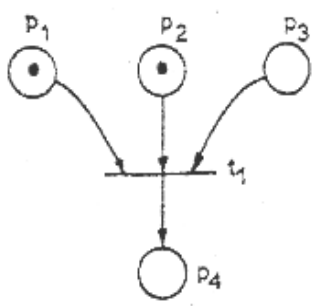

3(d) Synchronizetion

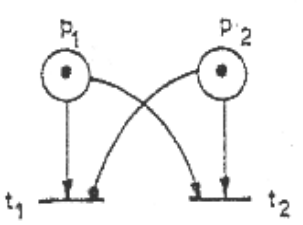

3(e) Frioritias

Figure 3. Petri Net Representation

Figure 3: GSNP Model of the Queuing Network 


\section{Representation Models for MSS.}

Our main result in this section is that for any MSS there exists a GSPN model that isomorphic to GSMP model as shown in the following theorem:

Theorem 4.1: Suppose that for any MSS there exists $\{X(t): t \geq 0\}$ that satisfies the conditions in Theorem 2.4 and $\{Y(t): t \geq 0\}$ that satisfies the conditions in Theorem 3.2. Then $X(t) \cong Y(t)$.

Proof: Assume that $S$ is the state space of $\{X(t): t \geq 0\}$ and $S^{*}$ is the state space of $\{Y(t): t \geq 0\}$. Define a measurable mapping $\psi$ from $S$ onto $S^{*}$ of the form

$$
\psi((s, E(s)))=(\lambda(s), \mu(E(s)))
$$

for any state $s$ in $X(t)$ where $\lambda, \mu$ are measurable mappings defined as follows:

$\lambda(s)=s^{*}$ (number of tokens per place) $\mu(E(s))=T\left(s^{*}\right) \subseteq T_{1} \cup T_{2}$ for any time $t$ and state $s$ in $X(t)$ since all mappings are assume to be onto, so that its clearly that $X(t)$ is isomorphic to $Y(t)$.

\section{Example 4.2 (Producer/Consumer Model)}

In the following example, we illustrate the use of the GSPN framework for formal specification of a MSS. In the graphical representation of a GSPN, places are drawn as circles, immediate transitions as thin bars, and timed transitions as thick bars, directed arcs connect transitions to output places and normal input places to transitions. Tokens are drawn as black dots

Consider a system consisting of two producers, two consumers, two buffers, and a single shared channel for transmission of items from buffer to consumers; [7]. Producer i creates items for consumer $i$ one at a time and items created, but not yet consumed, are placed in buffer $i$, $i=1,2$. Set

$X(t)=\left(U_{1}(t), U_{2}(t), V(t)\right)$, where $U_{\mathrm{i}}(t)$ denotes the number of items waiting in buffer $i$ and at time $t$ and

$V(t)=\left[\begin{array}{l}i \text { if the channel is transmitting an item to consumer } i \text { at time } t \\ 0 \text { if no transmission is underway at time } t,\end{array}\right.$

Where $i=1,2$. Formal specification of the stochastic process $\{X(t): t \geq 0\}$ is a MSS with state space

$S=\left\{\left(u_{1}, u_{2}, v\right) \in\{0,1,2, \ldots . .\}^{2} \times\{0,1,2, \ldots\}: v>0\right.$ when ever $u_{1}+u_{2}>$ $0\}$ and events set $E=\left\{e_{0}, e_{1}, e_{2}\right\}$, where $e_{0}=$ " end of transmission" and $e_{1}=$ " creation of item by producer $i$ ", $i=1,2$.

For $s=\left(u_{1}, u_{2}, v\right) \in S$, event $e_{0}$ belongs to $E(s)$ if and only if $v>0$. Events $e_{1}$ and $e_{2}$ belong to $E(s)$ for all $s \in S$. 
The MSS model can also be specified as a GSPN with deterministic timed and immediate transitions; see Figure 4.

Let $D=\left\{d_{1}, d_{2}, \ldots, d_{7}\right\}$ be the set of places of the GSPN, $E=\left\{e_{0}, e_{1}, \ldots, e_{6}\right\}$ be the set of transitions, and $E=\left\{e_{2}, e_{5}\right\}$ be the set of immediate transitions. Set $L\left(e_{5}\right)=\left\{d_{2}\right\}$ and $L\left(e_{1}\right)=1$ otherwise. Also set $I\left(e_{2}\right)=\left\{\mathrm{d}_{2}, d_{7}\right\}, I\left(e_{5}\right)=\left\{d_{5}, d_{7}\right\}$, and $\mathrm{I}\left(e_{\mathrm{j}}\right)=\left\{d_{\mathrm{j}}\right\}$, otherwise. Finally, set $J$ $\left(e_{1}\right)=\left\{d_{1}, d_{2}\right\}, J\left(e_{3}\right)=J\left(e_{6}\right)=\left\{d_{7}\right\}, J\left(e_{4}\right)=\left\{d_{4}, d_{5}\right\}$, and $J\left(e_{\mathrm{j}}\right)=\left\{d_{\mathrm{j}+1}\right\} ;$ otherwise.

The transitions have the following interpretation: $e_{\mathrm{j}}=$ "creation of item by producer 1 ", $e_{2}=$ "start of transmission from buffer 1 ", $e_{3}=$ "end of transmission from buffer 1", $e_{4}=$ "creation of item by producer 2", $e_{5}=$ "start of transmission from buffer $2 "$, and $e_{6}=$ "end of transmission from buffer $2 "$. The interpretation of the places is as follows.

There is always one token in place $d_{1}$ [respectively, place $d_{4}$ ] representing creation of items by producer 1 [respectively, producer 2]. There is a token in place $d_{3}$ [respectively, place $d_{6}$ ] if and only if the channel is transmitting an item to consumer 1 [respectively, consumer 2]; otherwise, there are no tokens in place $d_{3}$ [respectively, place $d_{6}$ ]. There are $(\mathrm{k} \geq 0)$ tokens in place $d_{2}$ [respectively, place $d_{5}$ ] if and only if there are $k$ items in buffer 1 [respectively, buffer 2] waiting to be transmitted. There is one token in place $d_{7}$ if and only if no item is being transmitted; otherwise, there are no tokens in place $d_{7}$. The marking set $G$ is $G=\left\{\left(s_{1}\right.\right.$, $\left.\left.s_{2}, \ldots, s_{7}\right) \in(\{1\} \times\{0,1, . .\} \times\{0,1\})^{2} \times\{0,1\}: s_{3}+s_{6}+s_{7}=1\right\}$

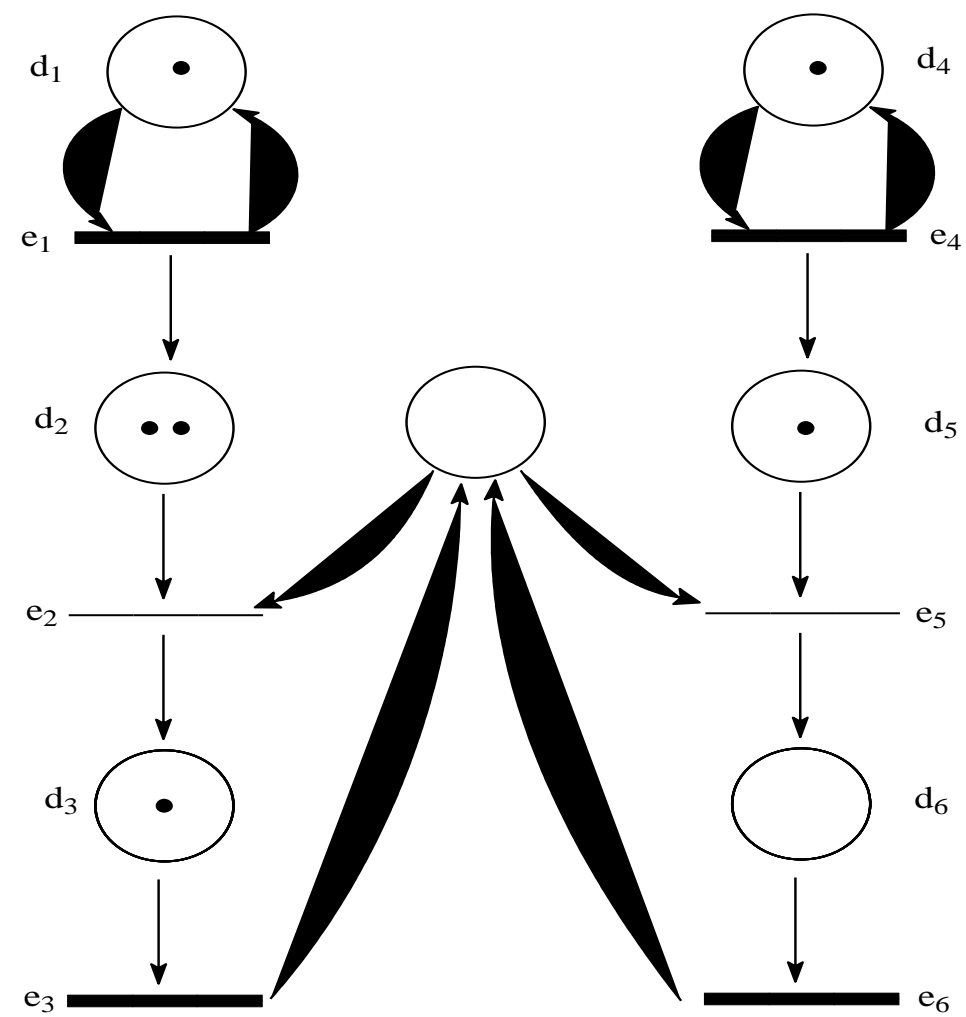

Figure 4. GSPN representation of producer/consumer mode1, see [7]. 


\section{CONCLUSION}

1- Since GSPN and GSMP models captures the dynamic structure of MSS (Theorem 4.1) allows us to study the stochastic behavior of MSS.

2- Since no analytical results are available for calculating the parameter selected to be study (For example the waiting time parameter) in the MSS, simulation is a natural recourse and the choosing of the adequate model (GSPN or GSMP) play important role because depend in the nature of the system and parameters selected to be study.

3- Since the steady - state in GSMP is give by abstract form while in the GSPN model the steady - state is given by graphical form therefore this property in GSPN is better than GSMP in some real Multiprocessor Stochastic System.

4- The isomorphism in (theorem 4.1) can be applied by using semimarkov or markov processes by putting $E(s)=\{e\}$ or $E(s)=\phi$ respectively.

\section{REFERENCES}

1) Ajmone Marsan, M., (1995). Modeling with Generalized stochastic petri Nets. john Wiley Series Parallel Computing-Chichester .

2) Crane, M. A. and Lemoine, A. J., (1977). An Introduction to the Regenerative Method for Simulation Analysis. Springer-Verlag Berlin - Heidelberg.

3) Dingle, N. J., Harrison, P.G. and Knottenbenbelt, W. J., (2011). Response Time Densities in Generalized Stochastic Petri Net Models. Preprint.

4) Bolch, G., Bruzsa, C. and Sztrik, J., (2011). Modelling and Simulation of Markov Modulated Multiprocessor Stochastic Systems with Petri Nets. Preprint .

5) Haas, P. J. and Shedler, G. S., (1987). Regenerative Generalized Semi - Markov process. Comm. Statist. Stochastic models 3, 409438.

6) Haas, P. J. and Shedler, G. S., (1987). Stochastic Petri Net Representation of Discrete event Simulations, IBM Research Report RJ 5646 (57145).

7) Haas, P. J. and Shedler, G. S., (1988). Modeling Power of Stochastic Petri Nets for Simulations. Probability in the Engineering and and informational Sciences 2, 435-459. 
8) Heyman, D. P. and Sobel, M. J. (1982). Stochastic Models in Operations Research, Volume I. McGraw - Hill.

9) Kelling, C. (1993). A New Method to Determine the Initial Checkpoint of the Spectra Variance Analysis International conference on Acheu .

10) Marsan, M. A., Bbalbo, G. and Conte, G., (1984) A. Class of Generalized Stochastic Petri Nets for the Performance Evaluation of Multiprocessor Stochestic Systems', ACM Transactions on Computer Systems, Vol. 2, No. 2, PP. 93-122,.

11) Motameni, H., Movaghar, A., Siasifar, M., Montazeri, H. and Rezaei, A., (2008). Analytic Evaluation Petri Net by Using Markov Chain Theory to Achieve Optimized Models. World Applied Sciences Journal 3(3): 504-513.

12) Narahari, Y., Suryanarayanan, K. and Subba reddy, N. V. (1989). Discrete Event Simulation of Distributed Systems Using Stochastic Petri Nets. IEEE,622-625

13) Robert, G., Pettit, I. V. and Gomaa, H., (2000). Validation of Dynamic Behavior in UML using Colored Petri Nets. UML 2000 Dynamic Behavior workshop, New York, England.

14) Schassberger, R. (1978). Insensitivity of Steady State Distributions of Generalized Semi - Markov Processes. Adv. App1. Prob. 10. $836-851$.

15) Shedler, G. S. (1987). Regeneration and Networks of Queues. Springer-Verlag.

16) Woodside, C. M. and Li, Y. (1991), Performance Petri net analysis of Communication protocol software by delay-equivalent aggregation. In Procedings of the 4th International Workshop on Petri nets and Performance Models, Pages 64-73, Melbourne, Australia, 2-5 December. IEEE Computer Society Press. 